\title{
ES Research Square

\section{Clinical and Quality of Life Outcomes Among Ixekizumab -Treated Psoriasis Patients in a Real- World Setting: Results from a Single US Dermatology Referral Practice}

Craig Leonardi

Central dermatology

Rei Tao

ICON plc https://orcid.org/0000-0002-0591-2071

Solmaz Setayeshgar

ICON plc

Sisi Wang

ICON plc

Suzanne McMullen

ICON plc

Russel Burge

Eli Lilly and Company

Baojin Zhu

Eli Lilly and Company

William N Malatestinic ( $\nabla$ malatestinic_william_n@lilly.com )

Eli Lilly and Company

Research article

Keywords: Quality of Life Outcomes, Ixekizumab (IXE), Psoriasis, Dermatology

Posted Date: October 5th, 2020

DOl: https://doi.org/10.21203/rs.3.rs-61531/v1

License: @ (i) This work is licensed under a Creative Commons Attribution 4.0 International License.

Read Full License 


\section{Abstract}

Background: Psoriasis is a chronic immune-mediated inflammatory skin condition that has a significant negative impact on the physical, emotional, and psychosocial well-being of those affected. Ixekizumab (IXE) has demonstrated an early and high-level treatment response in the real-world setting. This study aimed to assess the rapidity and long-term disease severity and quality of life (QOL) outcomes among IXE-treated plaque psoriasis patients.

Method: A retrospective cohort study was conducted at a single US dermatology referral center. Medical charts were reviewed for adult psoriasis patients starting IXE (index date) between March 22, 2016, and February 28, 2018. Disease severity and QOL data were abstracted up to one-year pre-IXE initiation and up to 35 months post-IXE initiation. Static Physician Global Assessment (sPGA), Body Surface Area (BSA), and Dermatology Life Quality Index (DLQI) were summarized at 1-month post-index and at 3month intervals. Logistic regressions were performed to evaluate the 1-month response in relation to long-term SPGA, BSA, DLQI outcomes. Subgroup analysis were conducted to compare outcomes among biologic naïve and biologic experienced patients.

Results: A total of 153 patients (median age at index: 47.7 years; $65.4 \%$ male; $93.5 \%$ Caucasian) were included in the study. Majority of patients $(69 \% ; n=106)$ were biologic experienced prior to IXE initiation. In the total study cohort, at 1 -month post-index $58.8 \%$ of patients achieved SPGA $(0,1), 55.9 \%$ achieved DLQI $(0,1)$, and $66.9 \%$ achieved BSA $\leq 1 \%$. Patients with sPGA $(0,1)$ at 1 -month post-index had greater odds of remaining sPGA $(0,1)$ and $B S A \leq 1 \%$ at 24 -month (sPGA 0,1: $O R=10.1 ; 95 \% \mathrm{Cl}: 2.1-47.9 ; \mathrm{BSA} \leq 1 \%$ : $\mathrm{OR}=13.3 ; 95 \% \mathrm{Cl}: 2 \cdot 2-80.2)$. Among patients who achieved SPGA $(0,1)$ at 1-month post-index, the proportion of patients with SPGA $(0,1)$, DLQI $(0,1)$, and BSA $\leq 1 \%$ remained largely the same for the 24month follow-up. Among biologic experienced and naïve patients, more than half achieved SPGA $(0,1)$, DLQI $(0,1)$, and $B S A \leq 1 \%$ at 1 -month after IXE initiation.

Conclusion: This real-word study demonstrated that majority of patients initiating IXE achieved sPGA ( 0 , $1)$, DLQI $(0,1)$ and BSA $\leq 1 \%$ targets within the first month of treatment and were able to maintain treatment response for up to 24 months independent of prior biologic exposure.

\section{Background}

Psoriasis is a chronic immune-mediated inflammatory skin condition that has a significant negative impact on the physical, emotional, and psychosocial well-being of those affected.(1) (2) Plaque psoriasis is the most common type of psoriasis accounting for approximately $90 \%$ psoriasis cases.(3) Chronic psoriasis is characterized by recurrent flares, or the sudden and severe worsening of symptoms, often without any obvious cause. In the United States (US), the estimated prevalence of psoriasis is $3.2 \%$ among individuals aged 20 years or older with greater prevalence found among Caucasians $(2.5 \%)$ compared to African Americans $(1.3 \%) .(4,5)$ 
The Psoriasis Area and Severity Index (PASI) is considered the gold standard measurement for psoriasis severity, yet it is rarely used in routine clinical practice due to its complexity.(6) Instead, physicians routinely quantify the percentage of body surface area (BSA) affected, use the 7-point static Physician Global Assessment (SPGA) scale, and use patient perception of impairment to different aspects of their health-related quality of life (HRQOL) in order to understand disease activity and severity.(6) The Dermatology Life Quality Index (DLQI) is a validated instrument which indicates the degree of reduction in patient's HRQoL. $(1,7)$

There is currently no cure for psoriasis, however, based on the severity of the disease, treatments are available to help manage symptoms. These include topical agents, phototherapy, conventional systemic therapies, biologics and over-the-counter therapies. The treatment paradigm for psoriasis tends towards the use of topical agents for mild and moderate disease, systemic agents for moderate and severe disease, and biologics for the more severe cases of psoriasis. ${ }^{(8)}$ Current available biologic therapies are inhibitors of T lymphocytes and cytokines: TNFa, IL-12, IL-23 and IL-17A, and these have shown efficacy in reducing psoriasis symptoms in moderate to severe cases. $(9,10)$ While rapid response and long-term effectiveness were indicated to be of high importance among patients with moderate to severe disease as well as physicians making treatment decisions, $(2,11)$ there is evidence that current biologic treatments may lose effectiveness over time. ${ }^{(12),(13,14)}$

The newer biologic therapies are those that inhibit the IL-17 or IL-23 pathways. Ixekizumab (Taltz®, IXE), an IL-17 antagonist, was approved in the US on March 22, 2016 for the treatment of adults with moderate-to-severe plaque psoriasis who are candidates for systemic therapy or phototherapy. $(15,16)$ IXE has demonstrated desired effectiveness in clinical trials $(17,18)$ and in real-world clinical practice, $(9$, 19) however, its speed of onset and long-term impact on patient outcomes and treatment persistence has not yet been assessed in a real-world setting.

The aims of this study are two-fold. First, to assess speed of IXE effectiveness and describe the persistency of outcomes among psoriasis patients at a single dermatology referral practice in the US. Second, to assess clinical and HRQoL outcomes in subgroups of patients who had a sPGA score of 0,1 at 1-month or were exposed to biologic treatment prior to IXE initiation. Findings from this study will provide foundational insights that can be used to inform future broader comparative effectiveness studies of IXE.

\section{Methods}

\section{Study design}

A retrospective cohort study was conducted at a single US dermatology referral center. Medical chart data were used to identify patients who initiated IXE between March 22, 2016 and February 28, 2018 (i.e. the enrolment period). The index date was defined as the date of IXE initiation. Data up to 35 months after index date and 12 months before index date were collected. The end of study period was defined as February 28, 2019, date of IXE discontinuation, or discontinuation of clinical care, whichever occurred 
first. All data collected were de-identified, and the study adhered to the Guidelines for Good Pharmacoepidemiology Practices(20) as well as the Health Insurance Portability and Accountability Act (HIPAA) guidelines for protection of patient confidentiality.(21)

\section{Data source and study population}

Data were collected from Central Dermatology, a dermatology referral practice encompassing one primary investigator supported by nurse practitioners. Patients with moderate to severe psoriasis are routinely seen at one month and then every three months during active treatment stage after biologic initiation for up to one year; and are seen approximately every six months beyond one year of treatment. SPGA, BSA, and DLQI are regularly collected at clinic visits.

Study population included adult patients with physician-diagnosed plaque psoriasis who initiated IXE during the enrolment period. Patients who received IXE by participating in any clinical trials for their psoriasis before or during the enrolment period as well as patients under the age of 18 at the time of IXE initiation were excluded from the study. Medical records of psoriasis patients who initiated treatment with IXE during the enrolment period were sampled consecutively in chronological order, based on IXE initiation date. Sampled charts were then screened against study eligibility criteria. The study was reviewed and approved by Advarra institutional review board (IRB), and a waiver of informed consent was granted.

\section{Data collection}

The medical records of psoriasis patients who met the eligibility criteria were retrospectively reviewed, data were abstracted up to one year prior to IXE initiation and up to 35 months post IXE initiation using an electronic data collection form (eDCF) created with Microsoft ${ }^{\circledR}$ Access. Patients were seen at different schedules depending on the course of their disease.

\section{Variables}

Baseline demographic and clinical information including age, sex, ethnicity, insurance status, and time since psoriasis diagnosis was obtained from medical records at index date. Use of systemic, topical and phototherapy treatments as well as treatment discontinuation, and reason for discontinuation were collected during the pre-index period. Primary treatment outcomes of interest collected during post-index were SPGA, DLQI, and BSA. SPGA is a 7-point scale that classifies the severity of psoriasis from " 0 = clear" to "6 = severe". DLQI responses were classified into five categories $(0-1,2-5,6-10,11-20$, and 21-30) from "no effect" to "extremely large effect".(6) BSA was presented as continuous variables and also categorised using National Psoriasis Foundation (NPF) guidelines as: treat to target (BSA $\leq 1 \%$ ), mild ( $1 \%$ $<\mathrm{BSA}<3 \%$ ), moderate ( $3 \% \leq \mathrm{BSA} \leq 10 \%)$, and severe (BSA $>10 \%$ ). (22) These outcomes as well as the specific body regions involved were collected during the post-index period for each documented visit.

\section{Statistical analysis}


Analyses were performed based on available patient data at each clinic visit. Descriptive analysis was used to summarize baseline demographic and clinical characteristics, treatment details, and study outcomes. Mean, standard deviation (SD), median, and range were summarized for continuous variables, and counts and percentages were summarized for categorical variables.

Disease severity measures were summarized from index date up to 24 months post-index. SPGA, DLQI, BSA and specific body regions involved were summarized by counts and percentages at 1-month postindex, at 3-month intervals for up to 12 months post-index, and at 6-month intervals in the second year following IXE initiation among patients with available data at each post-index time point (+/-45 days). Percentage of BSA and number of body regions involved were summarized by mean, SD, median, and range. Additionally, two subgroup analyses were performed: 1) comparing early responders, defined as patients who had achieved a sPGA $(0,1)$ at 1-month with those who did not; and 2) comparing patients who had used biologic systemic treatment prior to IXE initiation with patients who were biologic naïve.

Unadjusted logistic regressions were performed to explore the potential determinants of SPGA, DLQI, and BSA outcomes at 12 and 24 months after IXE initiation. The outcomes were BSA $\leq 1 \%$ versus BSA $>1 \%$, $\operatorname{SPGA}(0,1)$ versus $\mathrm{SPGA}>1$, and DLQI $(0,1)$ versus DLQI $>1$. Odds ratios $(\mathrm{OR})$ were presented alongside $95 \% \mathrm{Cl}$. Analyses were performed in SAS 9.4 (SAS Institute, Inc., Cary, NC).

\section{Results}

A total of 153 psoriasis patients treated with IXE between March 22, 2016 and February 28, 2018 and who met the study eligibility criteria were included in the study. Among patients seen at each individual study follow-up visit, nearly all patients had SPGA (98\%), BSA (99\%), and DLQI (96\%) documented. The median age at IXE-initiation was 47.7 years (range: $19.7-74.5$ ), $65.4 \%$ of the study population was male, and $93.5 \%$ of patients were Caucasian (Table 1 ). The median time since psoriasis diagnosis was 11.9 years (range: $0.1-41.1$ ). A total of 106 patients received prior biologic therapy for psoriasis; adalimumab was the most commonly used biologic prior to IXE initiation $(n=40)$, followed by secukinumab $(n=35)$. Almost half of the study cohort received prior non-biologic systemic treatment $(n=$ $69,45.1 \%)$ with the majority receiving methotrexate $(n=66,43.1 \%) .38 .6 \%(n=59)$ patients received topical treatment during the pre-index period while only two patients (1.3\%) received phototherapy. Among patients who switched to IXE from other biologic therapies, the majority of the patients discontinued their prior biologics due to inadequate response to treatment (TNF-a inhibitor: $90.8 \%$, IL12/23 inhibitor: $100 \%$, IL-17 inhibitor: $82.9 \%$, Table 2) 
Table 1

Baseline demographic and clinical characteristics

\section{Demographic characteristics}

Age at study index date (years)

Mean (SD)

Median (Min-Max)

Unknown

Sex, $n(\%)$

Male

Female

Race/ethnicity, n (\%)

Caucasian

African-American

Multi-racial

Unknown

Insurance status at study index date, n (\%)

Private

Medicaid

Medicare

Medicare;Private

None

Time since psoriasis diagnosis (years)*

Mean (SD)

Median (Min-Max)

Unknown

Treatment during pre-index**

Non-biologic systemic medications, $\mathbf{n}(\%)$

Methotrexate

Cyclosporine

Biologic systemic medications, $\mathbf{n}(\%)$
All patients $(n=153)$

$47.2(13.1)$

$47.7(19.7-74.5)$

$0(0.0)$

$100(65.4)$

$53(34.6)$

$143(93.5)$

5 (3.3)

$1(0.7)$

$4(2.6)$

$143(93.5)$

$1(0.7)$

$3(2.0)$

4 (2.6)

2 (1.3)

14.8 (10.5)

$11.9(0.1-41.1)$

34 (22.2)

69 (45.1)

66 (43.1)

3 (2.0)

106 (69.3) 


\begin{tabular}{|ll|}
\hline Demographic characteristics & All patients $(\mathbf{n}=\mathbf{1 5 3})$ \\
\hline TNF-a inhibitor & $66(43.1)$ \\
\hline Etanercept & $2(1.3)$ \\
\hline Infliximab & $9(5.9)$ \\
\hline Adalimumab & $40(26.1)$ \\
\hline Certolizumab & $8(5.2)$ \\
\hline Ampremilast & $9(5.9)$ \\
\hline IL-12/23 inhibitor & $14(9.2)$ \\
\hline $\begin{array}{l}\text { Ustekinumab } \\
\text { IL-17 inhibitor }\end{array}$ & $14(9.2)$ \\
\hline $\begin{array}{l}\text { Secukinumab } \\
\text { Topical medications, } \mathbf{n} \text { (\%) }\end{array}$ & $35(22.9)$ \\
\hline $\begin{array}{l}\text { Phototherapy, } \mathbf{n} \text { (\%) } \\
\text { Abbreviations: IL = Interleukin; } \mathrm{n}=\text { Number; SD = Standard deviationAbbreviations: TNF = }\end{array}$ \\
$\begin{array}{l}\text { Tumor necrosis factor } \\
* \text { The study index date is the date of ixekizumab initiation } \\
\star * \text { Patients may have received more than one treatment concomitantly or sequentially during pre-index } \\
\text { period; number of treatments } \\
\text { received can be higher than number of patients that received the treatment }\end{array}$ \\
\hline
\end{tabular}


Table 2

Reasons for treatment choice and treatment discontinuation of biologics in the pre-index period

\begin{tabular}{|c|c|c|c|}
\hline Reasons ${ }^{\wedge}$ & $\begin{array}{l}\text { TNF-a inhibitor ( } \mathrm{n} \\
=66)^{*}\end{array}$ & $\begin{array}{l}\text { IL-12/23 inhibitor } \\
(\mathrm{n}=14)^{*}\end{array}$ & $\begin{array}{l}\text { IL-17 inhibitor (n } \\
=35)^{*}\end{array}$ \\
\hline & n (\%) & n (\%) & n (\%) \\
\hline \multicolumn{4}{|l|}{ Treatment discontinuation } \\
\hline $\begin{array}{l}\text { Treatment ongoing at end of pre- } \\
\text { index period }\end{array}$ & $1(1.5)$ & $0(0.0)$ & $0(0.0)$ \\
\hline Treatment switch/discontinuation & $65(98.5)$ & $14(100.0)$ & 35 (100.0) \\
\hline \multicolumn{4}{|l|}{$\begin{array}{l}\text { Reason for treatment } \\
\text { switch/discontinuation }\end{array}$} \\
\hline Inadequate response to treatment & $59(90.8)$ & $14(100.0)$ & $29(82.9)$ \\
\hline Adverse event & $2(3.1)$ & $0(0.0)$ & $1(2.9)$ \\
\hline Insurance coverage & $1(1.5)$ & $0(0.0)$ & $1(2.9)$ \\
\hline Patient preference & $2(3.1)$ & $0(0.0)$ & $1(2.9)$ \\
\hline Doctor preference & $26(40.0)$ & $4(28.6)$ & $15(42.9)$ \\
\hline Side effect profile of biologic & $1(1.5)$ & $0(0.0)$ & $0(0.0)$ \\
\hline Other & $10(15.4)$ & $0(0.0)$ & $0(0.0)$ \\
\hline \multicolumn{4}{|c|}{ Abbreviations: IL = Interleukin; $\mathrm{n}=$ Number; TNF = Tumor necrosis factor } \\
\hline \multicolumn{4}{|c|}{$\begin{array}{l}{ }^{\wedge} \text { Patients may have had more than one reason for treatment choice or discontinuation or switch; } \\
\text { number of reasons can be higher than number of patients receiving treatment. }\end{array}$} \\
\hline \multicolumn{4}{|c|}{ *Denominator: total number of patients receiving the treatment. } \\
\hline \multicolumn{4}{|c|}{$\sim$ Denominator for this variable is number of pateints who discontinue the biologics. } \\
\hline
\end{tabular}

\section{Speed of treatment effect}

Disease severity during follow-up was summarized (Fig. 1). At index, $7.2 \%, 20.9 \%$, and $19.6 \%$ of patients had SPGA $(0,1)$, DLQI $(0,1)$, and BSA $\leq 1 \%$, respectively. By 1-month post-index $(n=136), 58.8 \%$ of patients achieved clear or almost clear skin (sPGA 0,1), 29.4\% clear skin (sPGA 0), 55.9\% DLQI $(0,1)$ and $66.9 \%$ BSA $\leq 1 \%$. The proportion of patients achieving sPGA $(0,1)$, DLQI $(0,1)$, and BSA $\leq 1 \%$ (NPF target) during follow-up are shown in Fig. 1. At 3-month post-index, nearly three-quarters $(73.5 \%)$ of patients had SPGA $(0,1)$, while $68.4 \%$ had DLQI $(0,1)$, and $86 \%$ had BSA $\leq 1 \%$. The median change during 24 months post-index period was approximately $3 \%$ reduction for BSA, -3 points for sPGA, and -2 points for DLQI scores for the overall study cohort. 
Subgroup analysis was done on early responders (defined as achieving SPGA 0, 1 at 1 month) and nonearly responders (not achieving SPGA $(0,1)$ at 1 month). Among 135 patients with 1-month post-index sPGA data available, $59.2 \%(n=80)$ of patients were early responders, among which $70 \%(n=56)$ achieved DLQI $(0,1)$, and $85 \%(n=68)$ achieved BSA $\leq 1 \%$. Over $85 \%, 75 \%$, and $65 \%$ of patients met the targets for BSA, SPGA, and DLQI, respectively, at all the time points after 1-month post-index among earlyresponders (Fig. 2). Among non-early responders $(n=55), 46.8 \%, 55.3 \%$, and $74.5 \%$ of them achieved the targets for SPGA, DLQI, and BSA at 3-month post-index, respectively. The proportions remained largely the same for 24-month post-index (Fig. 2).

Additional subgroup analyses were done among biologic naïve patients and biologic experience patients prior to index date (Fig. 2). Of the 109 patients who received biologic treatments for psoriasis at any time prior to IXE initiation, $6.5 \%$ had SPGA $(0,1), 25.7 \%$ had DLQI $(0,1)$, and $22 \%$ had BSA $\leq 1 \%$ at IXE initiation. 95 patients in the biologic experienced group had data available at 1-month post-index, and $58.9 \%$ had SPGA $(0,1)$ including $28.4 \%$ with SPGA $0 ; 55.8 \%$ of the patients achieved DLQI $(0,1)$; and $66.3 \%$ achieved BSA $\leq 1 \%$. At 3 -month, the proportions of patients achieving SPGA, DLQI and BSA targets increased to $72.6 \%$ (52.6\% patients had SPGA 0 ), $68.4 \%$, and $83.2 \%$, respectively. Of 44 biologic naïve patients, 41 patients had data available at 1- and 3-months post-index. The proportions of patients achieving sPGA, DLQI, and BSA targets were $58.5 \%$ and $75.7 \%, 68.3 \%$ and $75.7 \%, 68.3 \%$ and $92.7 \%$, respectively at 1 - and 3 -months.

\section{Long-term treatment effect}

Disease severity and quality of life outcomes at 24-month post-index were available for 49 patients from the overall study cohort. Among the 49 patients, $75.5 \%, 69.4 \%$, and $83.7 \%$ had treatment outcomes of $\operatorname{sPGA}(0,1)$, DLQI $(0,1)$, and BSA $\leq 1 \%$, at 24-month post index respectively. Among early responders, $87.9 \%$ of patients had SPGA $(0,1)$, including $66.7 \%$ with sPGA $0 ; 75.8 \%$ patients had DLQI $(0,1)$; and $93.9 \%$ BSA $\leq 1 \%$ at 24 month.

Logistics regression model results showed that at 24 months post-index, early sPGA responders at 1month not only had greater odds of achieving $\operatorname{sPGA}(0,1)(\mathrm{OR}=10.1 ; 95 \% \mathrm{Cl}: 2.1-47.9 ; \mathrm{p}$-value $=0.003)$, but also greater odds of achieving $B S A \leq 1 \%(O R=13.3 ; 95 \% \mathrm{Cl}$ : $2.2-80.2$; $p$-value $=0.005)$ than non-early responders. No association was found for DLQI $(0,1)$ at 24 months. Also, early SPGA responders were more likely to achieve the SPGA $(0,1)$ outcome at 12 months than non-early responders $(\mathrm{OR}=4.4 ; 95 \% \mathrm{Cl}$ : $1.7-11.1 ; p$-value $=0.002$, data not shown).

\section{Discussion}

This is the first observational study reporting 1- to 24-month real-world severity and quality of life outcomes following IXE initiation among patients with moderate-to-severe psoriasis in a US referral clinic. The current study showed that in the real-world setting, a high level of effectiveness can be observed as early as 1-month following IXE initiation and maintained for up to 24 months. We found that the majority of patients met the treatment targets of $\operatorname{SPGA}(0,1)$, DLQI $(0,1)$ and $B S A \leq 1 \%$ at 1 -month after initiating 
IXE. Most patients not achieving SPGA $(0,1)$ after 1-month of IXE treatment were still able to achieve response of SPGA $(0,1)$ by 3 months. Most of the study cohort were on a prior biologic before switching to IXE with mean treatment duration of 9.8 months (SD: 6.7 months). Despite inadequate response to previous biologics, more than half of patients achieved a rapid response at 1-month after IXE initiation, and the proportion of patients meeting treatment targets increased at 3-month and remained relatively stable onward.

The current study found that the majority of patients from the overall study cohort as well as in the subgroups of early responders and non-early responders all maintained high levels of responses of sPGA, DLQI, and BSA outcomes throughout the 24-month follow-up period. These findings were consistent with the 5-year results from the UNCOVER-1 and 2 studies, reported by Leonardi et al, in which clinical and quality of life responses of IXE were maintained throughout the 5-year period. $(23,24)$ Similar rapid response results were found in other real-world studies outside the US, where significant improvement in disease severity measured by PASI was observed at one-month after IXE initiation and continued to improve at 3-month and at 6-months. $(9,19), 28$ In the current study, BSA percentage at IXE initiation and a rapid response to IXE at one month were found to be strongly associated with the 24-month IXE treatment outcomes in the study.

The key strength of the study was the availability of long-term clinical and DLQI data collection, which enables the investigation of long-term treatment outcomes, as well as the frequency of outcomes collected at each time point. In the current study, a third $(n=49)$ of the study cohort had 24-month postindex data, and treatment outcomes were collected at 1-month and as frequently as every 3 months during the first year following IXE initiation. This density of follow-up visits is not available from other real-world data sources. For example, the CORRONA registry, established in April 2015 to collect prospective real-world data on patients with autoimmune diseases, including those with psoriasis who are treated with biologic therapies in the US and Canada only collects data on clinical and patient reported outcomes (i.e. BSA, SPGA) at six-month intervals.(25) This makes it difficult to assess speed of treatment response. This single-arm retrospective chart review study was able to address this knowledge gap. Additionally, the outcomes reported in our study were measured by a single physician at study site which limit the inter-rater variations. Other strengths of this study include the use of a consecutive sampling approach based on treatment initiation date to reduce selection bias, and the unique study design that allows the collection of both pre-index data of up to 12 months and post-index data of up to 35 months. Such study design allows the exploration of treatment history and its impact on subsequent IXE treatment outcomes. The overall patient population and results in this study were consistent to clinical trials,(26) and the two real-world studies conducted in Europe and one in Canada which used PASI as disease severity measure. $(9,19)^{28}$

There are several limitations to the current study. First, a single study site was chosen based on the availability of relevant data at the site, however, efforts were made to randomly select charts within the stratifications of interest, and the findings of this study are expected to reflect the real-world outcomes of the target patient population. Second, this study looked at cross-sectional clinical and HRQoL data at 
each follow-up time point. Although the use of patient medical records offers may potential advantages over other data sources such administrative data, in research studies that require detailed clinical information,(27) the use of retrospective data has the inherent limitation of having been collected for purposes independent of the study objectives. The level of detail available depends largely on the charting practices of the study site. For the changes in disease severity and HRQoL analysis performed in this study, only patients with available data at each study time point were used; therefore, the sample size declined over time due to infrequent clinic visits or losses to follow-up. The regression analyses performed in the study were unadjusted due to this sample size constraint, and further investigations on the strength of these results are needed in larger study populations.

In conclusion, the findings of the current real-world study support the speed of response and persistency of effect seen in IXE clinical trials. $(23,24)$ Results from this study demonstrated that the majority of patients achieved SPGA $(0,1)$, DLQI $(0,1)$ and BSA $\leq 1 \%$ targets within the first month of treatment which were also achieved at each follow-up time point up to 24 months. Findings from this study provide valuable information on speed of treatment response and long-term outcomes in IXE-treated psoriasis patients and in other subgroup populations.

\section{List Of Abbreviations}

\begin{tabular}{|ll|}
\hline Abbreviation & Full description \\
\hline BSA & Body surface area \\
\hline CM & Centimetre \\
DLQI & Electronic data collection form \\
\hline HRQoL & Dermatology Life Quality Index \\
IL & Health-related quality of life \\
\hline IXE & Interleukin \\
NPF & Ixekizumab \\
SPGA & National Psoriasis Foundation \\
PASI & Static Physician Global Assessment \\
\hline TNF & Psoriasis Area and Severity Index \\
\hline
\end{tabular}

\section{Declarations}

Ethics approval and consent to participate 
Ethical approval was obtained from Advarra, Inc., prior to beginning data collection. A Waiver of informed was granted.

All analyses and reporting were carried out using de-identified data. This study adhered to the Guidelines for Good Pharmacoepidemiology Practices ${ }^{(20)}$ as well as the Health Insurance Portability and Accountability Act (HIPAA) guidelines for protection of patient confidentiality. ${ }^{(21)}$

\section{Consent for publication}

Not applicable, the manuscript does not contain individual person's data

\section{Availability of data and materials}

The datasets used and/or analysed during the current study are available from the corresponding author on reasonable request.

\section{Competing interests}

C. Leonardi has received honoraria from Eli Lilly for the following: Advisory Board, Speaker, and Consultant.

RT, SS, SW, SM are employees of ICON plc., a company contracted by Eli Lilly to conduct this study.

RB, BZ, and WNM are employees of Eli Lilly.

\section{Funding}

The study was funded by Eli Lilly and Company.

\section{Author's contributions}

CL: conception of the study.

WNM, RT, SW: conception of the study, design of the study.

SS: Statistical analysis.

All authors involved in interpretation of data and critical revision of the work.

All authors read and approved the final manuscript.

\section{Acknowledgements}

Not applicable

\section{References}


1. Guenther L, Langley RG, Shear NH, Bissonnette R, Ho V, Lynde C, et al. Integrating biologic agents into management of moderate-to-severe psoriasis: a consensus of the Canadian Psoriasis Expert Panel. February 27, 2004. J Cutan Med Surg. 2004;8(5):321-37.

2. Gorelick J, Shrom D, Sikand K, Renda L, Burge R, Dworkin C, et al. Understanding Treatment Preferences in Patients with Moderate to Severe Plaque Psoriasis in the USA: Results from a CrossSectional Patient Survey. Dermatology and Therapy. 2019;9(4):785-97.

3. Sarac G, Koca TT, Baglan T. A brief summary of clinical types of psoriasis. North Clin Istanb. 2016;3(1):79-82.

4. Rachakonda TD, Schupp CW, Armstrong AW. Psoriasis prevalence among adults in the United States. Journal of the American Academy of Dermatology. 2014;70(3):512-6.

5. Gelfand JM, Stern RS, Nijsten T, Feldman SR, Thomas J, Kist J, et al. The prevalence of psoriasis in African Americans: Results from a population-based study. Journal of the American Academy of Dermatology. 2005;52(1):23-6.

6. Feldman SR, Krueger GG. Psoriasis assessment tools in clinical trials. Ann Rheum Dis. 2005;64 Suppl 2:ii65-ii8.

7. Finlay AY, Khan GK. Dermatology Life Quality Index (DLQI)-a simple practical measure for routine clinical use. Clin Exp Dermatol. 1994;19(3):210-6.

8. DiPiro J, Talbert R, Yee G, ea. Pharmacotherapy: A Pathophysiologic Approach (8th ed.) Mc-Graw-Hill Medical; 2011.

9. Chiricozzi A, Burlando M, Caldarola G, Conti A, Damiani G, De Simone C, et al. Ixekizumab Effectiveness and Safety in the Treatment of Moderate-to-Severe Plaque Psoriasis: A Multicenter, Retrospective Observational Study. American journal of clinical dermatology. 2019.

10. Bilal J, Berlinberg A, Bhattacharjee S, Trost J, Riaz IB, Kurtzman DJB. A systematic review and metaanalysis of the efficacy and safety of the interleukin (IL)-12/23 and IL-17 inhibitors ustekinumab, secukinumab, ixekizumab, brodalumab, guselkumab and tildrakizumab for the treatment of moderate to severe plaque psoriasis. The Journal of dermatological treatment. 2018;29(6):569-78.

11. Alcusky M, Lee S, Lau G, Chiu GR, Hadker N, Deshpande A, et al. Dermatologist and Patient Preferences in Choosing Treatments for Moderate to Severe Psoriasis. Dermatology and Therapy. 2017;7(4):463-83.

12. Hsu L, Snodgrass BT, Armstrong AW. Antidrug antibodies in psoriasis: a systematic review. The British journal of dermatology. 2014;170(2):261-73.

13. Rompoti N, Sidiropoulou P, Panagakis P, Stratigos A, Papoutsaki M, Stefanaki E, et al. Real world data from a single Greek center on the use of secukinumab in plaque psoriasis: effectiveness, safety, drug survival, and identification of patients that sustain optimal response. Journal of the European Academy of Dermatology and Venereology : JEADV. 2020.

14. Smith JA, Wehausen B, Richardson I, Zhao Y, Li Y, Herrera V, et al. Treatment Changes in Patients With Moderate to Severe Psoriasis: A Retrospective Chart Review. Journal of cutaneous medicine and surgery. 2018;22(1):25-30. 
15. FDA. FDA approves new psoriasis drug Taltz: U.S. Food \& Drug Administration; 2016 [Available from: https://www.fda.gov/NewsEvents/Newsroom/PressAnnouncements/ucm491872.htm.

16. Eli Lilly. Taltz-ixekizumab injection, solution. Addendalndex Summary 2017 [Available from: http://uspl.lilly.com/taltz/taltz.html\#pi.

17. Gordon KB, Colombel JF, Hardin DS. Phase 3 Trials of Ixekizumab in Moderate-to-Severe Plaque Psoriasis. N Engl J Med. 2016;375(21):2102.

18. Sawyer LM, Cornic L, Levin LA, Gibbons C, Moller AH, Jemec GB. Long-term efficacy of novel therapies in moderate-to-severe plaque psoriasis: a systematic review and network meta-analysis of PASI response. Journal of the European Academy of Dermatology and Venereology : JEADV. 2019;33(2):355-66.

19. Deza G, Notario J, Lopez-Ferrer A, Vilarrasa E, Ferran M, Del Alcazar E, et al. Initial results of ixekizumab efficacy and safety in real-world plaque psoriasis patients: a multicentre retrospective study. Journal of the European Academy of Dermatology and Venereology : JEADV. 2019;33(3):5539.

20. Ispe. Guidelines for good pharmacoepidemiology practices (GPP). Pharmacoepidemiol Drug Saf. 2008;17(2):200-8.

21. The Health Insurance Portability and Accountability Act of 1996 (HIPPA). 1996.

22. Menter A, Strober BE, Kaplan DH, Kivelevitch D, Prater EF, Stoff B, et al. Joint AAD-NPF guidelines of care for the management and treatment of psoriasis with biologics. Journal of the American Academy of Dermatology. 2019;80(4):1029-72.

23. Leonardi C, Maari C, Philipp S, Goldblum O, Zhang L, Burkhardt N, et al. Maintenance of skin clearance with ixekizumab treatment of psoriasis: Three-year results from the UNCOVER-3 study. Journal of the American Academy of Dermatology. 2018;79(5):824-30.e2.

24. Leonardi C, Reich K, Foley P, Torii H, Gerdes S, Guenther L, et al. Efficacy and Safety of Ixekizumab Through 5 Years in Moderate-to-Severe Psoriasis: Long-Term Results from the UNCOVER-1 and UNCOVER-2 Phase-3 Randomized Controlled Trials. Dermatol Ther (Heidelb). 2020.

25. Corrona. Corrona Registries: Psoriasis. 2017.

26. Leonardi C, Reich K, Foley P, Torii H, Gerdes S, Guenther L, et al. Efficacy and Safety of Ixekizumab Through 5 Years in Moderate-to-Severe Psoriasis: Long-Term Results from the UNCOVER-1 and UNCOVER-2 Phase-3 Randomized Controlled Trials. Dermatology and Therapy. 2020;10(3):431-47.

27. Liang S-Y, Phillips KA, Wang G, Keohane C, Armstrong J, Morris WM, et al. Tradeoffs of using administrative claims and medical records to identify the use of personalized medicine for patients with breast cancer. Med Care. 2011;49(6):e1-e8.

\section{Figures}


Figure 1

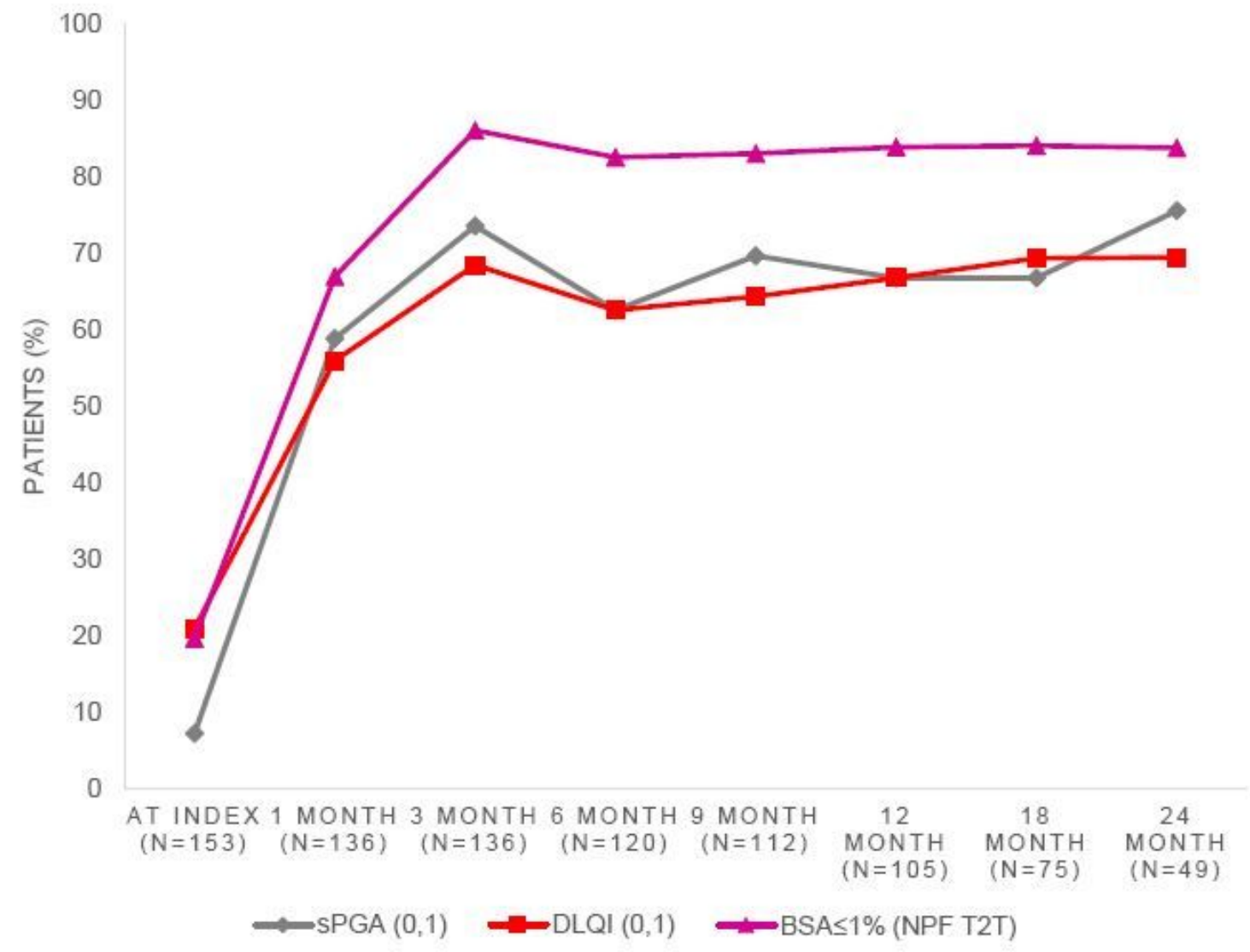

Figure 1

Percentage of patients achieving treatment targets during 24-month post-index, among overall study cohort $(n=153)$ 
Figure 2.
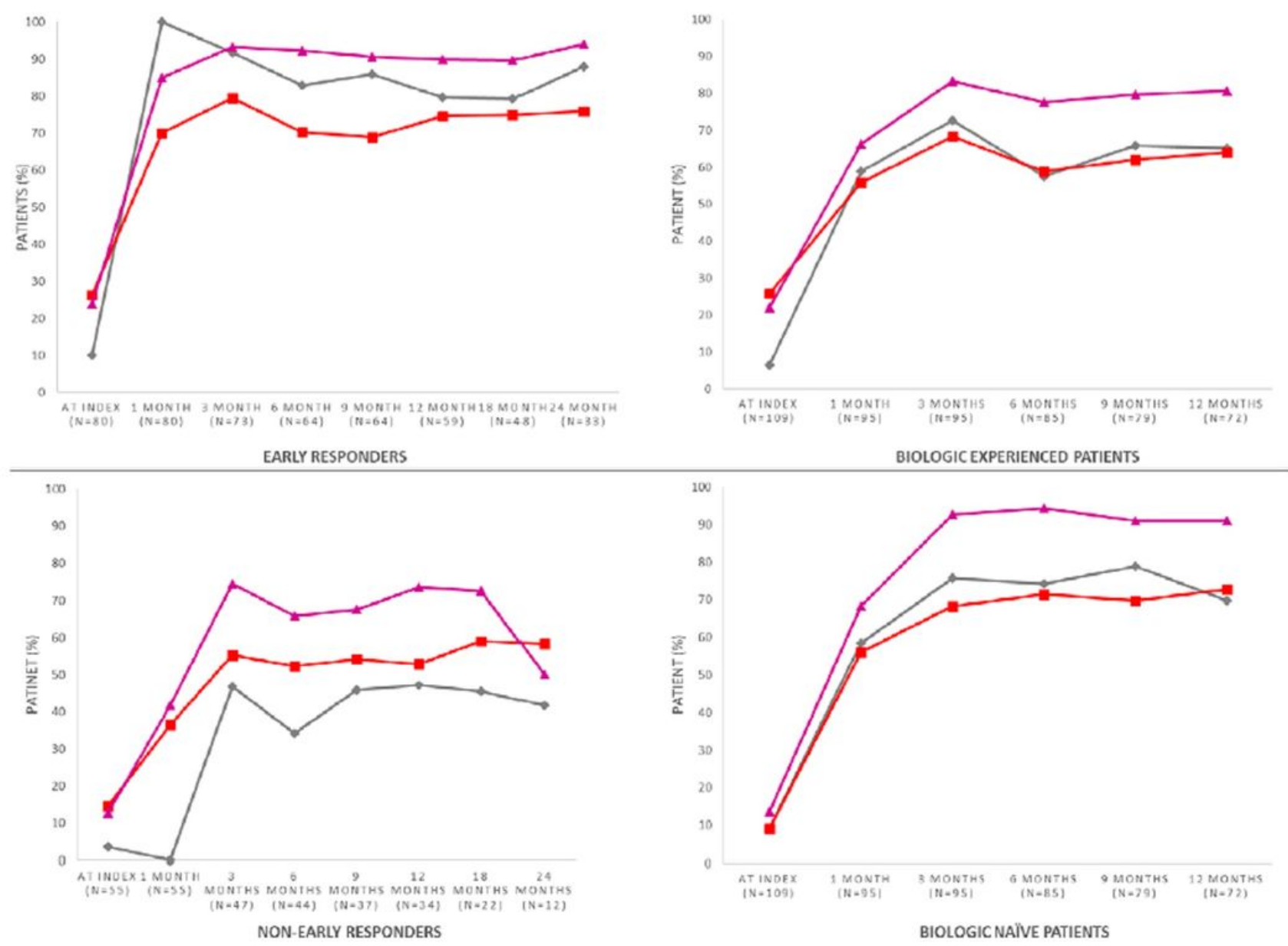

\section{Figure 2}

Percentage of patients achieving treatment targets during post-index among subgroups 